\title{
Platelet mass index as a novel inflammatory marker for tinnitus
}

\author{
Ahmet Doblan ${ }^{1}$ and togay müderris ${ }^{2}$ \\ ${ }^{1}$ Sanliurfa Mehmet Akif Inan Egitim ve Arastirma Hastanesi \\ ${ }^{2}$ Izmir Bozyaka Egitim ve Arastirma Hastanesi
}

November 27, 2020

\begin{abstract}
Abstract: Although there is plenty of research on the etiology and treatment of tinnitus, a definitive conclusion has not been reached in most studies. Among etiological factors, vascular and hematological abnormalities can be defined as an important cause in the evaluation of tinnitus cases, and these factors may underly many structural and functional problems. In this study, we aimed to investigate whether there was a correlation between platelet mass index (PMI) and tinnitus. To our knowledge, this is the first study exploring the effect of platelet mass on tinnitus. After reviewing the detailed medical records of 1,079 tinnitus patients that presented to our clinic between January 2019 and May 2020, the hemogram values of 177 cases meeting the study criteria were evaluated. There was no statistically significant difference between the patient and control groups in terms of mean age, gender distribution, and RBC (Red Blood Cell) neutrophil, lymphocyte, PLR and NLR levels ( $\mathrm{p}>0.05$ ). Compared to the control group, the hemoglobin and MPV levels of the patient group were statistically significantly higher $(\mathrm{p}<0.001)$, and the platelet level was statistically significantly lower $(\mathrm{p}=0.033)$. In addition, the PMI level of the patient group was significantly higher than that of the control group $(\mathrm{p}<0.001)$. In conclusion, the NLR, PLR and MPV values were found to be higher in the tinnitus group compared to the control group, as expected. Furthermore, the additional data for tinnitus cases showed that PMI was also observed to be significantly higher compared to the control group, indicating that the study objective was reached. PMI seems to be a predictive value for tinnitus, even in patients with MPV values lower than the normal range. Therefore, we consider that PMI is more sensitive than other markers, especially MPV in inflammatory events.
\end{abstract}

\section{Platelet mass index and tinnitus}

Abstract:

Although there is plenty of research on the etiology and treatment of tinnitus, a definitive conclusion has not been reached in most studies. Among etiological factors, vascular and hematological abnormalities can be defined as an important cause in the evaluation of tinnitus cases, and these factors may underly many structural and functional problems. In this study, we aimed to investigate whether there was a correlation between platelet mass index (PMI) and tinnitus. To our knowledge, this is the first study exploring the effect of platelet mass on tinnitus.

After reviewing the detailed medical records (examination notes, drug use, radiological evaluations, audiological evaluations, and previous surgical procedures) of 1,079 tinnitus patients that presented to our clinic between January 2019 and May 2020, the hemogram values of 177 cases meeting the study criteria were evaluated. Age, gender, hemoglobin, neutrophil, lymphocyte, platelet, and mean platelet volume (MPV) were examined. This was followed by the assessment of the distributions of age, gender, hemoglobin level, neutrophil lymphocyte ratio (NLR), platelet lymphocyte ratio (PLR), and PMI.

There was no statistically significant difference between the patient and control groups in terms of mean age, gender distribution, and RBC (Red Blood Cell), neutrophil, lymphocyte, PLR and NLR levels ( $\mathrm{p}>0.05$ ). Compared to the control group, the hemoglobin and MPV levels of the patient group were statistically 
significantly higher $(\mathrm{p}<0.001)$, and the platelet level was statistically significantly lower $(\mathrm{p}=0.033)$. In addition, the PMI level of the patient group was significantly higher than that of the control group $(\mathrm{p}<0.001)$.

In conclusion, the NLR, PLR and MPV values were found to be higher in the tinnitus group compared to the control group, as expected. Furthermore, the additional data for tinnitus cases showed that PMI was also observed to be significantly higher compared to the control group, indicating that the study objective was reached. PMI seems to be a predictive value for tinnitus, even in patients with MPV values lower than the normal range. Therefore, we consider that PMI is more sensitive than other markers, especially MPV in inflammatory events.

Key words: Tinnitus, Platelet Mass Index, NLR, PLR, MPV

What's already known about this topic?

-mpv is a inflamatory marker. And there are some study about this subject. also hematological parameters have an important place in tinnitus etiology. Here we studied the most sensitive marker among all hematological data in this study.

What does this article add?

- 1- PMI is a more sensitive indicator than MPV

- A cutt off value is set for PMI

- Can show a way to treat tinnitus

Review criteria: how did you gather, select and analyze the information you considered in your review?

-Medical records of all patients diagnosed with tinnitus was retrospectively reviewed.

-we excluded all diseases that cause tinnitus.

Message for the clinic: what is the 'take-home' message for the clinician?

-The function and number of platelets are as important as the volume.

- PMI can express them all together.

-PMI is the parameter that most clearly demonstrates the role of platelets in tinnitus.

\section{Introduction:}

Tinnitus is hearing sounds of different frequencies and qualities in the ear without any external stimulus [1]. Tinnitus is evaluated in two main groups as objective and subjective. Objective tinnitus is mostly caused by temporomandibular joint disorder, arteriovenous malformations, and spasm of the tensor timpani muscle, and in this form, the person performing the test hears the sound together with the patient [2]. In subjective tinnitus, the sound is only heard by the patient, not the person conducting the test. Subjective tinnitus may develop for many reasons, such as hearing impairment, insomnia, medication use, depression, chronic diseases, anxiety, and difficulty concentrating, and it has a negative impact on the quality of life of patients [3]. Tinnitus can have various frequencies and qualities, as well as various intensities. It can reach disturbing levels that seriously affect the daily life and social performance of the sufferer.

Despite the abundance of studies on the etiology and treatment of tinnitus, most do not present definitive conclusions. However, it is a known fact that although the majority of tinnitus sounds heard are considered to originate from the cochlea, tinnitus continues even after the auditory nerve has been completely resected [4]. This situation shows that tinnitus can originate not only from the cochlea but also from all auditory pathways, and can be seen after the formation of some inappropriate neural plasticities in the central nervous system. Although these changes in the central nervous system are both structural and functional, they can be seen in different parts of the brain, as well as the auditory pathways [5, 6]. Among all these factors, vascular and hematological abnormalities can be defined as an important etiology when tinnitus cases are 
evaluated, and there may even be underlying many structural and functional problems. In sudden hearing loss and facial paralysis, the vascular and hematological dimensions of tinnitus have been the subject of many studies, but a clear result has not yet been determined. Among these possible hematological causes, the neutrophil to lymphocyte ratio (NLR) and platelet to lymphocyte ratio (PLR) have been associated with the inflammatory process and some thrombotic events [7]. The effect of these parameters on tinnitus has been investigated in many publications, and results have been reported based on different levels of evidence. However, considering that the main function in thrombosis risk is related to thrombocytes, they should be examined more carefully. In these thrombotic events, the platelet mass index (PMI), which is considered to be very effective at the microvascular level, comes to the fore. This is especially important in organs with endarteria, such as the ear cochlea. Any microvascular thrombotic event caused by platelets in these endarteria causes serious problems in organ functions. This situation cannot always be revealed by angiographic methods and it is vital to consider the possibility of transient ischemic attacks, and thus tissue damage. In addition, in the literature, it has been emphasized that the increase in the mean platelet volume (MPV) is effective in inflammatory processes and that increased MPV values are directly related to the activation of platelets [8]. Although MPV is a parameter that has been previously investigated, its evaluation alone may not be sufficiently reliable since the number of platelets is at least as important as their volume. Therefore, in this study, we aimed to explore whether there was a correlation between PMI and tinnitus. To our knowledge, this is the first study investigating the effect of platelet mass on tinnitus.

\section{Material and method:}

After obtaining the approval of the ethics committee of Harran University, dated June 29, 2020 and numbered HRU/20.12.09, the detailed medical records (examination notes, drug use, radiological evaluations, audiological evaluations, and previous surgical procedures) of 1,079 tinnitus patients presenting to our clinic between January 2019 and May 2020 were examined, and the hemogram data of 177 cases meeting the inclusion criteria were analyzed. Following a detailed evaluation of medical records, patients with chronic tinnitus (continuing for at least three months) in the 18-60 age group were included in the study. In line with the medical records, cancer patients, patients with chronic disease, those with a history of cardiac or vascular surgery in the head and neck region, and those with platelet dysfunction were excluded from the study to determine the independent effect of platelet mass. Further excluded were patients with acute inflammation or infection, diabetes mellitus, systemic hypertension, hyperlipidemia, coronary artery disease, chronic liver disease, acute or chronic renal failure, chronic obstructive pulmonary disease, connective tissue disease, inflammatory bowel disease or a history of acoustic trauma, current smokers, patients with a personal or family history of hearing loss due to noise, those with a hearing loss above $20 \mathrm{~dB}$ caused by any otological disease, such as chronic otitis media, otosclerosis and Meniere's disease, and those with chronic diseases. For the patients included in the study, age, gender, hemoglobin, neutrophil, lymphocyte, platelet, and MPV were examined. This was followed by the assessment of the distribution of age, gender and hemoglobin level, NLR, PLR, and PMI (calculated as platelet number $\mathrm{x}$ MPV/1000). The values of the tinnitus group were compared with the data of 192 patients without a history of temporary (lasting less than two weeks) or permanent tinnitus. The control group consisted of patients daily presenting to the outpatient clinic for any reason other than otologic complaints. Cancer patients with chronic diseases, those with a history of cardiac or vascular surgery in the head and neck region, and those with platelet dysfunction were not included in this group. The medical history of this group were evaluated at the same sensitivity as the tinnitus group in terms of chronic diseases.

\section{Statistical Analysis}

Data analysis was performed using IBM SPSS Statistics software version 17.0 (IBM Corporation, Armonk, NY, USA). Whether the distributions of continuous variables were normal was determined using the Kolmogorov-Smirnov test. The assumption of the homogeneity of variances was examined with the Levene test. Descriptive statistics for continuous variables were expressed as mean \pm standard deviation values or median $\left(25^{\text {th }}-75^{\text {th }}\right.$ percentiles), where appropriate. Numbers and percentages were used for categorical data. The mean differences between the groups were compared using Student's t-test when the parametrical 
test assumptions were met, and the Mann-Whitney U test otherwise. Categorical data were analyzed using Pearson's $\chi^{2}$ test. The optimal cut-off points of laboratory measurements to discriminate cases and controls were evaluated with the ROC (Receiver Operating Characteristic) analysis by obtaining the maximum sum of sensitivity and specificity for the significant test. The sensitivity, specificity, positive and negative predictive values, and accuracy levels for PMI were also calculated. A p value less than 0.05 was considered as statistically significant.

Results:

Table 1 presents a comparison of the demographic characteristics and laboratory measurements of the control and tinnitus groups. There was no statistically significant difference between the control and tinnitus groups concerning the mean age, gender and RBC, neutrophil, lymphocyte, PLR and NLR levels ( $\mathrm{p}>0.05)$. However, in the tinnitus group, the hemoglobin and MPV levels were statistically significantly higher $(\mathrm{p}<$ 0.001), and the PLT level was statistically significantly lower $(\mathrm{p}=0.033)$ compared to the control group. In addition, the PMI of the tinnitus group was statistically significantly higher than that of the control group $(\mathrm{p}<0.001)$ (see Figure 1).

Table 2 shows the results of the ROC analysis conducted to determine whether PMI, PLR and NLR were statistically significant markers in distinguishing between the control and tinnitus groups. The area under the curve (AUC) for the PMI measurements was found to be statistically significant in this differentiation $[$ AUC $=0.678,95 \%$ CI: $0.623-0.732$ and $\mathrm{p}<0.001$ ] (see Figure 2). The optimal cut-off value for PMI was 1.6452 , at which PMI had a sensitivity of $89.8 \%$, specificity of $40.5 \%$, and positive and negative predictive values of $58.3 \%$ and $81.1 \%$, respectively, and the diagnostic accuracy rate of this parameter was $64.2 \%$.

The areas under the ROC curve for the PLR and NLR measurements in differentiating between the control and tinnitus groups were not found to be statistically significant $(\mathrm{AUC}=0.539,95 \%$ CI: $0.479-0.598$ and $\mathrm{p}$ $=0.201$ and $\mathrm{AUC}=0.521,95 \% \mathrm{CI}: 0.462-0.580$ and $\mathrm{p}=0.485$, respectively). In other words, neither PLR nor NLR has a statistically significant effect on this differentiation.

Discussion:

Tinnitus is a disease in which the patient reports hearing sounds of different character and intensity coming from the ear without any external stimulus. This condition results in a large number of patients presenting to otorhinolaryngology clinics. Although 10-15\% prevalence has been reported in different case series, this rate may exceed $30 \%$ in the population over 60 years of age [9]. Tinnitus can affect one ear or both ears. There are many studies on the role of platelets in this condition. In our study, we tried to determine the rate of real vascular causes by excluding factors leading to temporary tinnitus, such as fatigue, insomnia, short-term drug use, and advanced age (presbycusis) in order to reveal the independent effect of vascular and hematological variables. It is known that MPV is directly related to inflammatory processes and platelet function [8]. Increased MPV has been identified as an independent risk factor for myocardial infarction and stroke [10]. Therefore, the presence of various effects of mediators secreted by platelets on tissues, especially neovascularization is a fact that should not be overlooked. Considering only the volume of platelets and evaluating them regardless of their number will lead to a lack of interpretation concerning platelet function. However, it is also important to recognize that the significant difference in MPV may be due to pre-measurement factors, including blood collection tube selection, processing and equipment used [11]. Increased MPV indicates an increase in granulocytes in platelets, which is directly related to their activity; i.e., larger platelets are more active than smaller platelets [12].

In addition to the volumetric size of platelets, their number is also important. Ischemia causes an inflammatory process to begin. In ischemic tissues, many proinflammatory cytokines, mainly TNF-alpha, are released in the affected tissue [13]. It is considered that an ischemic situation in auditory pathways caused by vascular pathologies may trigger a mechanism that results in an increase in MPV. This increase in MPV or platelet count naturally leads to an increase in PMI. In our study, NLR and PLR were found to be higher in the tinnitus patients than in the control group, indicating the association of these parameters with inflammatory and ischemic events, similar to many previous publications. In a study by Kemal et al., MPV was observed to 
be increased in tinnitus patients, and the authors suggested that tinnitus might occur as a result of increased MPV causing microvascular thrombosis in the vessels of the auditory tract and hypoperfusion-ischemia [14]. However, we consider that it is not appropriate to consider MPV alone to explain all these mechanisms. MPV is a very practical and inexpensive parameter that can be measured as part of a routine complete blood count analysis. However, various factors, such as age, obesity, hyperlipidemia, diabetes, smoking, and hypertension can affect platelet parameters [8]. Thus, the overall platelet function can be more accurately reflected by platelet mass compared to platelet count alone. This idea was first discussed by Gerday et al., who demonstrated that with the use of PMI, the number of platelet transfusions decreased [15].

To the best of our knowledge, this is the first study to explore the relationship between PMI and tinnitus in the literature. We consider that PMI, in which platelet counts are also evaluated, will be more useful than MPV alone in explaining the mechanism of tinnitus. In animal experiments, the antioxidant effects of platelets have been shown to reduce oxidant-induced pulmonary edema by preventing increased membrane permeability [16]. Platelets maintain normal endothelial permeability in lungs exposed to ischemia-reperfusion injury by releasing platelet glutathione-redox cycle antioxidants [17]. Lo et al. also reported an increase in pulmonary vascular permeability in animals with thrombocytopenia [18]. Some studies on platelet count in neonatal lung tissues have revealed its effect on inflammatory processes. PMI is associated with platelet functionality since larger platelets are enzymatically more active than smaller platelets. Recently, some studies aimed to reduce unnecessary transfusion by using PMI rather than platelet count as an indicator of the need for this procedure $[15,19]$. For all these reasons, PMI is considered to be a better marker than MPV in some cases. Okur et al. reported that premature babies with bronchopulmonary dysplasia, intraventricular hemorrhage, necrotizing enterocolitis, retinopathy of prematurity, and sepsis had lower PMI in the early period compared to those without these neonatal morbidities. The authors noted that PMI was significantly lower in these neonatal complications although the differences in MPV were not significant [20]. This situation may also be valid for tinnitus patients.

In our study, we found that PMI was statistically significantly higher in the tinnitus group, but larger series are needed to prove the superiority of PMI over MPV in tinnitus pathogenesis. Although there were patients with lower MPV values in the tinnitus group than in the control group, the fact that PMI was higher in the former suggests that it is a more sensitive parameter. It was even the case that the MPV value was very similar and almost equal for certain cases in the two groups whereas PMI was consistently higher in the tinnitus group. When these findings are analyzed one by one, they each indicate that PMI may be a more accurate and sensitive parameter than MPV in the etiopathogenesis of the disease and for use as a clinical marker in tinnitus patients. In our evaluation, we did not take the severity of tinnitus into account nor did we administer any questionnaire (e.g., Tinnitus Handicap Inventory) to the tinnitus patients for this purpose. This is because we did not have enough evidence that vascular causes affect the severity of tinnitus. The significant difference we detected in PMI between the tinnitus and control groups can guide further studies to investigate the relationship between tinnitus severity and PMI.

A high neutrophil and platelet count reflects inflammation while a low lymphocyte count is indicative of general stress and impaired defense mechanisms [21]. In otolaryngology practice, high NLR values have been found to be associated with a variety of pathological conditions, such as vestibular neuritis [22], Bell's palsy [23], idiopathic sudden sensorineural hearing loss [24], and squamous epithelial cell carcinoma of the head and neck [25]. Recently, Özbay et al. [26] reported significantly higher NLR values in patients with severe tinnitus than controls and concluded that NLR should be considered as a potential clinical marker of tinnitus. From this point of view, it is seen that NLR has an important place in the evaluation of tinnitus. In our study, we determined NLR values consistent with previous research and confirmed that they were significantly different in tinnitus patients compared to controls. We also observed a positive correlation between PMI and NLR.

PLR, another marker of inflammatory processes, has been used together with NLR in previous literature studies. In particular, higher PLR values have been reported in vestibular neuritis and Bell's palsy compared to control groups, and in this respect, PLR has been accepted as an important parameter [22, 23]. Some 
studies even argue that PLR is a more significant inflammation marker than NLR and provides more information concerning prognosis. For example, Turkmen et al. [27] stated that it could predict inflammation better than NLR in patients with end-stage renal disease. PLR was also found to be superior to NLR in predicting clinical outcome in patients with soft tissue carcinoma [28]. However, due to the lack of a detailed study on tinnitus, such a comparison cannot yet be made for this condition. Although we do not have data on the superiority of PLR or NLR over each other, when both NLR and PLR are considered as inflammation markers, they are reported to be correlated with each other in literature studies, as was the case in our research.

Limitations:

The retrospective design was the greatest disadvantage of this study, causing a serious limitation in the number of patients and the necessity to keep the exclusion criteria very wide; thus, our patient sample group was limited. In addition, the inclusion of tinnitus severity scoring in a further study conducted in line with our results can provide data that we were not able to present in the current study.

In conclusion, in this study, the NLR, PLR and MPV values were found to be higher in the tinnitus group compared to the control group, as expected. However, as additional data for tinnitus cases, PMI was also observed to be significantly higher compared to the control group, indicating that the study reached its objective. PMI has managed to become a predictive value for tinnitus even in patients with lower MPV levels than normal. Therefore, we consider that PMI is more sensitive than other markers, especially MPV in inflammatory events. We believe that PMI can be suitable and provide meaningful results in many areas in otolaryngology and should be further investigated in cases with Bells' palsy vestibular neuritis, nasal polyps and allergic rhinitis, and sudden sensorineural hearing loss.

*The authors declare no conflict of interest.

**The authors declare that they did not receive any financial support for the study.

References

1. Wegger, M., T. Ovesen, and D.G. Larsen, Acoustic coordinated reset neuromodulation: a systematic review of a novel therapy for tinnitus. Frontiers in neurology, 2017. 8 : p. 36.

2. Bauer, C.A., Tinnitus. New England Journal of Medicine, 2018.378 (13): p. 1224-1231.

3. Zeman, F., et al., Which tinnitus-related aspects are relevant for quality of life and depression: results from a large international multicentre sample. Health and Quality of Life Outcomes, 2014.12 (1): p. 7.

4. Kohno, M., et al., Prognosis of tinnitus after acoustic neuroma surgery-surgical management of postoperative tinnitus. World Neurosurgery, 2014. 81 (2): p. 357-367.

5. Husain, F.T. and S.A. Schmidt, Using resting state functional connectivity to unravel networks of tinnitus. Hearing research, 2014.307 : p. 153-162.

6. Adjamian, P., M. Sereda, and D.A. Hall, The mechanisms of tinnitus: perspectives from human functional neuroimaging. Hearing research, 2009. 253 (1-2): p. 15-31.

7. Ferroni, P., et al., Venous thromboembolism risk prediction in ambulatory cancer patients: clinical significance of neutrophil/lymphocyte ratio and platelet/lymphocyte ratio.International journal of cancer, 2015. 136 (5): p. 1234-1240.

8. Karli, R., et al., Mean platelet volume: is it a predictive parameter in the diagnosis of sudden sensorineural hearing loss? Indian Journal of Otolaryngology and Head \& Neck Surgery, 2013.65 (4): p. 350-353.

9. Tunkel, D.E., et al., Clinical practice guideline: tinnitus.Otolaryngology-Head and Neck Surgery, 2014. 151 (2_suppl): p. S1-S40. 
10. Sarıkaya, Y., et al., Increased mean platelet volume in patients with idiopathic subjective tinnitus. European Archives of Oto-Rhino-Laryngology, 2016. 273 (11): p. 3533-3536.

11. Noris, P., F. Melazzini, and C.L. Balduini, New roles for mean platelet volume measurement in the clinical practice? Platelets, 2016.27 (7): p. 607-612.

12. Vizioli, L., S. Muscari, and A. Muscari, The relationship of mean platelet volume with the risk and prognosis of cardiovascular diseases. International journal of clinical practice, 2009.63 (10): p. 1509-1515.

13. Daemen, M.A., et al., Inhibition of apoptosis induced by ischemia-reperfusion prevents inflammation. The Journal of clinical investigation, 1999. 104 (5): p. 541-549.

14. Kemal, O., et al., Prognostic value of mean platelet volume on tinnitus. The Journal of laryngology and otology, 2016.130 (2): p. 162.

15. Gerday, E., et al., Testing platelet mass versus platelet count to guide platelet transfusions in the neonatal intensive care unit. Transfusion, 2009. 49 (10): p. 2034-2039.

16. Heffner, J.E., J.A. Cook, and P.V. Halushka, Human platelets modulate edema formation in isolated rabbit lungs. The Journal of clinical investigation, 1989. 84 (3): p. 757-764.

17. Zamora, C.A., D. Baron, and J.E. Heffner, Washed human platelets prevent ischemia-reperfusion edema in isolated rabbit lungs.Journal of Applied Physiology, 1991. 70 (3): p. 1075-1084.

18. Lo, S., et al., Role of platelets in maintenance of pulmonary vascular permeability to protein. American Journal of Physiology-Heart and Circulatory Physiology, 1988. 254 (4): p. H763-H771.

19. Zisk, J.L., et al., Transfusing neonates based on platelet count vs. platelet mass: a randomized feasibilitypilot study.Platelets, 2014. 25 (7): p. 513-516.

20. Okur, N., et al., Platelet mass index in very preterm infants: can it be used as a parameter for neonatal morbidities? The Journal of Maternal-Fetal \& Neonatal Medicine, 2016. 29 (19): p. 3218-3222.

21. Tefferi, A., C.A. Hanson, and D.J. Inwards. How to interpret and pursue an abnormal complete blood cell count in adults . in Mayo Clinic Proceedings . 2005. Elsevier.

22. Chung, J.H., et al., The significance of neutrophil to lymphocyte ratio and platelet to lymphocyte ratio in vestibular neuritis. The Laryngoscope, 2015. 125 (7): p. E257-E261.

23. Bucak, A., et al., Neutrophil-to-lymphocyte ratio as a novel-potential marker for predicting prognosis of Bell palsy. The Laryngoscope, 2014. 124 (7): p. 1678-1681.

24. Seo, Y.J., J.Y. Choi, and I.S. Moon, Neutrophil-to-lymphocyte ratio and platelet-to-lymphocyte ratio: novel markers for diagnosis and prognosis in patients with idiopathic sudden sensorineural hearing loss. Disease markers, 2014. 2014 .

25. Rachidi, S., et al., Neutrophil-to-lymphocyte ratio and overall survival in all sites of head and neck squamous cell carcinoma.Head \& neck, 2016. 38 (S1): p. E1068-E1074.

26. Ozbay, I., et al., Neutrophil-to-lymphocyte ratio in patients with severe tinnitus: prospective, controlled clinical study. J Laryngol Otol, 2015. 129 (6): p. 544-7.

27. Turkmen, K., et al., Platelet-to-lymphocyte ratio better predicts inflammation than neutrophil-tolymphocyte ratio in end-stage renal disease patients. Hemodialysis International, 2013.17 (3): p. 391-396.

28. Que, Y., et al., Preoperative platelet-lymphocyte ratio is superior to neutrophil-lymphocyte ratio as a prognostic factor for soft-tissue sarcoma. BMC cancer, 2015. 15 (1): p. 648.

\section{Hosted file}


TABLE 1.pdf available at https://authorea.com/users/379455/articles/495649-platelet-massindex-as-a-novel-inflammatory-marker-for-tinnitus

\section{Hosted file}

TABLE 2.pdf available at https://authorea.com/users/379455/articles/495649-platelet-massindex-as-a-novel-inflammatory-marker-for-tinnitus

\section{Hosted file}

F\selectlanguage\{polish\}I\selectlanguage\{english\}G 1.pdf available at https://authorea.com/ users/379455/articles/495649-platelet-mass-index-as-a-novel-inflammatory-marker-fortinnitus

\section{Hosted file}

F\selectlanguage\{polish\}I \selectlanguage\{english\}G 2.pdf available at https://authorea.com/ users/379455/articles/495649-platelet-mass-index-as-a-novel-inflammatory-marker-fortinnitus 